Article

\title{
Functional Boundedness of Balleans: Coarse Versions of Compactness
}

\author{
Taras Banakh ${ }^{1,2 *(1)}$ and Igor Protasov ${ }^{3}$ (1) \\ 1 Faculty of Mechanics and Mathematics, Ivan Franko National University of Lviv, Universytetska, \\ 79000 Lviv, Ukraine \\ 2 Institute of Mathematics, Jan Kochanowski University, Świętokrzyska 15, 25-406 Kielce, Poland \\ 2 Faculty of Cybernetics, Taras Shevchenko National University, Glushkov avenue 4d, 03680 Kyiv, \\ Ukraine; i.v.protasov@gmail.com \\ * Correspondence: t.o.banakh@gmail.com; Tel.: +38-097-651-1234
}

Received: 9 February 2019; Accepted: 8 March 2019; Published: 14 March 2019

\begin{abstract}
We survey some results and pose some open problems related to boundedness of real-valued functions on balleans and coarse spaces. Special attention is paid to balleans on groups. The boundedness of functions that respect the coarse structure of a ballean could be considered as a coarse counterpart of pseudo-compactness.
\end{abstract}

Keywords: coarse structure; ballean; group; real-valued function; boundedness

\section{Introduction}

In this paper, we consider various classes of real-valued functions (bornologous, macro-uniform, eventually macro- uniform, boundedly oscillating, slowly oscillating) that respect the coarse structure of a ballean and study balleans on which every such function is bounded on the complement of some bounded set. The boundedness of functions with respect to the coarse structure of a ballean could be considered as a coarse counterpart of compactness (which is a very important notion in Topology).

Balleans are sets endowed with the ball structure. Ball structures are bases of coarse structures. Coarse structures play an important role in Geometric Theory of Groups (see ([1], Chapter 3) and ([2], Chapter 4)), where groups $G$ are studied as balleans endowed with the finitary ball structure $\mathcal{E}=\left\{E_{F}: F \in[G]^{<\omega}\right\}$ where $E_{F}=\{(x, y) \in G \times G: y \in\{x\} \cup F x\}$ for a finite subset $F$ of $G$. The finitary ballean of a group $G$ is a partial case of the $\kappa$-ballean where $\kappa$ is an infinite cardinal. The $\kappa$-ballean of a group $G$ consists of entourages $E_{F}=\{(x, y) \in G \times G: y \in\{x\} \cup F x\}$ parametrized by subsets $F \subset G$ of cardinality $|F|<\kappa$.

Various types of real-valued functions that respect the coarse structure, play an important role in studying balleans and coarse spaces. In particular, macro-uniform maps are natural morphisms between balleans; slowly oscillating functions are used to define the Higson compactification and the Higson corona of a ballean, see [3,4], ([5], Chapter 8), [6], §2.9. Many properties of the coarse structure of a ballean reflect in the topological properties of its Higson corona.

Section 2 is of preliminary character and contains the definitions of various types of real-valued functions on balleans, as well as the corresponding notions of boundedness of balleans.

In Section 3, we study various real-valued functions on discrete balleans and show (in Proposition 3) that a ballean $X$ is discrete if and only if (iff) every real-valued function on $X$ 
is slowly oscillating. Moreover, if no measurable cardinal exists, then ballean $X$ is discrete iff every real-valued function on $X$ is boundedly oscillating iff every real-valued function on $X$ is eventially macro-uniform. In Proposition 4, we characterize discrete balleans on which every slowly oscillating real-valued function is bounded on the complement of some bounded set (such balleans are called so-bounded).

In Section 4, we put and discuss some open problems on the behavior of functional boundedness under products.

In Section 5, we study the boundedness properties of $\kappa$-balleans of groups. In Theorem 3 , we prove that the $|G|$-ballean of infinite group $G$ of regular cardinality is not so-bounded, which meant that $G$ admitts a slowly oscillating function $f: G \rightarrow \mathbb{R}$, which is unbounded on the complement $G \backslash F$ of any subset $F \subset G$ of cardinality $|F|<|G|$. For groups of singular cardinality, the situation depends on the algebraic properties of the group. If a group $G$ is free, then its $|G|$-ballean is not so-bounded (see Theorem 4). On the other hand, the $|G|$-ballean of any Abelian group $G$ of singular cardinality is emu-bounded and hence so-bounded (see Theorem 7). Moreover, for any cardinal $\kappa<|G|$ of uncountable cofinality, the $\kappa$-ballean of an Abelian group $G$ is emu-bounded and so-bounded. This implies that for any Abelian group $G$ of cardinality continuum (for example, $G=\mathbb{R}$ ), the $\omega_{1}$-ballean of $G$ is so-bounded iff $\omega_{1}<\mathfrak{c}$ (i.e., if the Continuum Hypothesis fails).

In Section 5, we also characterize groups whose finitary ballean is mu-bounded and show that such groups are tightly related with Bergman and (almost) Shelah groups, studied by Bergman [7] and Shelah [8].

In spite of the obtained results, many interesting problems related to the boundedness of real-valued functions on coarse spaces remain open. Such problems are formulated in the corresponding sections of the paper.

\section{Preliminaries}

A ballean is a pair $\left(X, \mathcal{E}_{X}\right)$ consisting of a set $X$ and a family $\mathcal{E}_{X}$ of subsets of the square $X \times X$ satisfying the following three axioms:

1. Each $E \in \mathcal{E}_{X}$ contains the diagonal $\Delta_{X}=\{(x, x): x \in X\}$ of $X$.

2. for any $E, F \in \mathcal{E}_{X}$ there exists $D \in \mathcal{E}_{X}$, such that $E \circ F^{-1} \subset D$, where

$E \circ F:=\{(x, z): \exists y(x, y) \in E,(y, z) \in F\}$ and $F^{-1}:=\{(y, x):(x, y) \in F\}$.

3. $\cup \mathcal{E}_{X}=X \times X$.

The family $\mathcal{E}_{X}$ is called the ball structure of the ballean $\left(X, \mathcal{E}_{X}\right)$, and its elements are called entourages. For each entourage $E \in \mathcal{E}_{X}$ and point $x \in X$, we could consider the set $E[x]:=\{y \in X$ : $(x, y) \in E\}$, called the ball of radius $E$, centered at $x$. For a subset $A \subset X$, the set $E[A]:=\bigcup_{a \in A} E[a]$ is called the E-neighborhood of $A$. Since $E=\bigcup_{x \in X}\{x\} \times E[x]$, the entourage $E$ can be recovered from the family of balls $E[x], x \in X$.

For a ballean $\left(X, \mathcal{E}_{X}\right)$ and a subset $Y \subset X$, the ballean $\left(Y, \mathcal{E}_{X}\lceil Y)\right.$ is called a subballean of $X$ when endowed with the following ball structure:

$$
\mathcal{E}_{X} \uparrow Y:=\left\{(Y \times Y) \cap E: E \in \mathcal{E}_{X}\right\} .
$$

Any metric space $(X, d)$ carries a natural ball structure $\left\{E_{\varepsilon}: \varepsilon>0\right\}$ consisting of the entourages $E_{\varepsilon}:=\{(x, y) \in X \times X: d(x, y)<\varepsilon\}$.

A ballean $\left(X, \mathcal{E}_{X}\right)$ is called a coarse space if for any entourage $E \in \mathcal{E}_{X}$, any subset $F \subset E$ with $\Delta_{X} \subset F$ belongs to $\mathcal{E}_{X}$. In this case, $\mathcal{E}_{X}$ is called the coarse structure of $X$. For a coarse structure $\mathcal{E}$, a subfamily $\mathcal{B} \subset \mathcal{E}$ is called a base of $\mathcal{E}$ if each set $E \in \mathcal{E}$ is contained in some set $B \in \mathcal{B}$. It is easy 
to see that each base of a coarse structure is a ball structure. On the other hand, each ball structure $\mathcal{E}$ on a set $X$ is a base of the unique coarse structure:

$$
\downarrow \mathcal{E}:=\left\{E \subset X \times X: \Delta_{X} \subset E \subset F \text { for some } F \in \mathcal{E}\right\} .
$$

If the ball (or coarse) structure $\mathcal{E}_{X}$ is clear from the context, we shall write $X$ instead of $\left(X, \mathcal{E}_{X}\right)$. More information on balleans and coarse spaces can be found in [1,5,6,9-11].

A subset $B \subset X$ of a ballean $\left(X, \mathcal{E}_{X}\right)$ is called bounded if $B \subset E[x]$ for some $E \in \mathcal{E}_{X}$ and $x \in X$. A ballean $X$ is bounded if $X$ is a bounded set in $\left(X, \mathcal{E}_{X}\right)$.

The family $\mathcal{B}_{X}$ of all bounded subsets is called the bornology of the ballean $\left(X, \mathcal{E}_{X}\right)$. If the ballean $X$ is unbounded, then the bornology $\mathcal{B}_{X}$ is an ideal of subsets of $X$. A family $\mathcal{I}$ of subsets of a set $X$ is called an ideal on $X$ if $\mathcal{I}$ is closed under finite unions and taking subsets, and $X \notin \mathcal{I}$.

A real-valued function $f: X \rightarrow \mathbb{R}$ on a ballean $X$ is called:

- bornologous if $f(B)$ is bounded for each $B \in \mathcal{B}_{X}$;

- macro-uniform (or a mu-function) if for every $E \in \mathcal{E}_{X}$ there exists a real number $C$ such that $\operatorname{diam} f(E[x])<C$ for every $x \in X$;

- eventually macro-uniform (or an emu-function) if for every $E \in \mathcal{E}_{X}$ there exists a bounded set $B \in \mathcal{B}_{X}$ and a real number $C$ such that diam $f(E[x])<C$ for every $x \in X \backslash B$;

- boundedly oscillating (or a bo-function) if there exists a real number $C$ such that for every $E \in \mathcal{E}_{X}$ there is $B \in \mathcal{B}_{X}$ such that diam $f(E[x])<C$ for each $x \in X \backslash B$;

- slowly oscillating (or a so-function) if for every $E \in \mathcal{E}_{X}$ and $\varepsilon>0$, there exists $B \in \mathcal{B}_{X}$ such that $\operatorname{diam} f(E[x])<\varepsilon$ for each $x \in X \backslash B$; and

- constant at infinity if for every $\varepsilon>0$ there exists a bounded set $B \in \mathcal{B}_{X}$ such that $\operatorname{diam} f(X \backslash B)<\varepsilon$.

Here, for a subset $A$ of the real line, we put $\operatorname{diam} A=\sup (\{0\} \cup\{|x-y|: x, y \in A\})$. We say that a ballean $X$ is:

- $\quad b$-bounded if every bornologous function $f: X \rightarrow \mathbb{R}$ is bounded;

- $\quad m u$-bounded if every mu-function $f: X \rightarrow \mathbb{R}$ is bounded;

- emu-bounded if for every emu-function $f: X \rightarrow \mathbb{R}$ there exists $B \in \mathcal{B}_{X}$ such that $f$ is bounded on $X \backslash B$;

- bo-bounded if for every bo-function $f: X \rightarrow \mathbb{R}$ there exists $B \in \mathcal{B}_{X}$ such that $f$ is bounded on $X \backslash B$;

- so-bounded (or pseudobounded) if for every so-function $f: X \rightarrow \mathbb{R}$ there exists $B \in \mathcal{B}_{X}$ such that $f$ is bounded on $X \backslash B$.

For any ballean $X$, we would have the following implications:

b-bounded $\Longrightarrow$ mu-bounded $\Longleftarrow$ emu-bounded $\Longrightarrow$ bo-bounded $\Longrightarrow$ so-bounded

If a ballean $X$ is unbounded and $\mathcal{E}_{X}$ has a linearly ordered base, then $X$ is b-unbounded iff $X$ is mu-unbounded iff $\mathcal{E}_{X}$ has a countable base.

We say that a ballean $X$ is locally finite if $\mathcal{B}_{X}=[X]^{<\omega}$, equivalently, each ball in $X$ is finite. Here, for a set $X$ and a cardinal $\kappa$ by $[X]^{<\kappa}$, we denote the family of subsets $A \subset X$ of cardinality $|A|<\kappa$.

A locally finite ballean $X$ is emu-bounded iff $X$ is mu-bounded. If a locally finite ballean $X$ is bo-bounded (so-bounded), then every bo-function (so-function) on $X$ is bounded. 
Example 1. Let $\kappa, \mu$ be infinite cardinals such that $\kappa \leq \mu$. We denote by $S_{\kappa}$ the group of all permutations of $\kappa$ and, for each $F \in\left[S_{\kappa}\right]<\mu$, put $E_{F}=\left\{(x, y) \in \kappa \times \kappa: y \in\{x\} \cup\{f(x)\}_{f \in F}\right\}$. Then the family $\left\{E_{F}: F \in\left[S_{\kappa}\right]^{<\mu}\right\}$ forms a base for some coarse structure $\mathcal{E}$ on $\kappa$. We denote the ballean $(\kappa, \mathcal{E})$ by $B_{\kappa, \mu}$ and observe that a subset $B \subset \kappa$ is bounded iff $|B|<\mu$.

We show that $B_{\kappa, \mu}$ is emu-bounded (and hence mu-bounded).

First, we check that $B_{\kappa, \mu}$ is mu-bounded. Given any unbounded function $f: \kappa \rightarrow \mathbb{R}$, we shall prove that $f$ is not macro-uniform. We choose a sequence $\left(x_{n}\right)_{n \in \omega}$ in $\kappa$ such that $\mid f\left(x_{n+1}\right)-$ $f\left(x_{n}\right) \mid>n$ and define a bijection $h: \kappa \rightarrow \kappa$ by the rule $h\left(x_{2 n}\right)=x_{2 n+1}, h\left(x_{2 n+1}\right)=x_{2 n}$ and $h(x)=x$ for all $x \in X \backslash\left\{x_{n}: n \in \omega\right\}$. Then $\operatorname{diam} f\left(E_{\{h\}}\left[x_{n}\right]\right)>n$, which means that $f$ is not macro-unifiorm.

Next, we show that the ballean $B_{\kappa, \mu}$ is emu-bounded. Given an eventually macro-uniform function $f: \kappa \rightarrow \mathbb{R}$, we should find a set $B \subset \kappa$ of cardinality $|B|<\mu$ such that the set $f(\kappa \backslash B)$ is bounded. If $\mu=\omega$, then the eventually macro-uniform function $f$ is macro-uniform and $f$ is bounded by the mu-boundedness of $B_{\kappa, \mu}$. So, we assume that the cardinal $\mu$ is uncountable.

To derive a contradiction, assume that for any subset $B \subset \kappa$ of cardinality $|B|<\mu$, the set $f(\kappa \backslash B)$ is unbounded. By transfinite induction, for every ordinal $\alpha<\mu$, we can construct a sequence $\left\{x_{\alpha, n}\right\}_{n \in \omega} \subset \kappa$ such that $\left|f\left(x_{\alpha, n+1}\right)-f\left(x_{\alpha, n}\right)\right|>n$ and $x_{\alpha, n} \notin\left\{x_{\beta, m}: \beta<\alpha, m \in \omega\right\}$ for every $n \in \omega$. Consider a permutation $h \in S_{\kappa}$ such that $h\left(x_{\alpha, 2 n}\right)=x_{\alpha, 2 n+1}, h\left(x_{\alpha, 2 n+1}\right)=x_{\alpha, 2 n}$ for all $\alpha<\mu$ and $n \in \omega$. Since the sets $\left\{x_{\alpha, n}\right\}_{n \in \omega}, \alpha<\mu$, are pairwise disjoint, for any set $B \subset \kappa$ of cardinality $|B|<\mu$ there exists an ordinal $\alpha<\mu$ such that $B \cap\left\{x_{\alpha, n}\right\}_{n \in \omega}=\varnothing$. Then $\operatorname{diam} f\left(E_{\{h\}}\left[x_{\alpha, 2 n}\right]\right) \geq \operatorname{diam}\left\{f\left(x_{\alpha, 2 n+1}\right), f\left(x_{\alpha, 2 n}\right)\right\}>2 n$, which means that $f$ is not eventually macro-uniform. But this contradicts the choice of $f$. This contradiction implies that for some set $B \subset \kappa$ of cardinality $|B|<\mu$ the set $f(\kappa \backslash B)$ is bounded.

Let $(X, \mathcal{E}),\left(X^{\prime}, \mathcal{E}^{\prime}\right)$ be balleans. A mapping $f: X \rightarrow X^{\prime}$ is called macro-uniform if for every $E \in \mathcal{E}$ there is $E^{\prime} \in \mathcal{E}^{\prime}$ such that $f(E[x]) \subseteq E^{\prime}[f(x)]$ for all $x \in X$. A bijection $f: X \rightarrow X^{\prime}$ is called an asymorphism if $f, f^{-1}$ are macro-uniform. The balleans $(X, \mathcal{E}),\left(X^{\prime}, \mathcal{E}^{\prime}\right)$ are called coarsely equivalent if there exists a large subset $Y \subseteq X, Y^{\prime} \subseteq X$ such that the balleans $(Y, \mathcal{E}\lceil Y)$ and $\left(Y^{\prime}, \mathcal{E}^{\prime} \uparrow Y^{\prime}\right)$ are asymorphic. A subset $Y$ is called large if $X=E[Y]$ for some $E \in \mathcal{E}$.

It can be shown that all the notions of boundedness, as defined above, are stable under coarse equivalences.

By a bornology on a set $X$, we understand any family $\mathcal{B}$ of subsets of $X$ such that $\cup \mathcal{B}=X$ and $\mathcal{B}$ is closed under finite unions and taking subsets. In particular, for any infinite cardinal $\kappa$, the family

$$
[X]^{<\kappa}:=\{A \subset X:|A|<\kappa\}
$$

is a bornology on $X$.

A bornology $\mathcal{B}$ on a set $X$ is called tall if every infinite subset of $X$ has an infinite bounded subset. A bornology $\mathcal{B}$ is tall iff every bornologous function on $X$ is bounded. Hence, a ballean $X$ is b-bounded iff its bornology $\mathcal{B}_{X}$ is tall.

A bornology $\mathcal{B}$ on $X$ is called antitall if every unbounded subset of $X$ contains an unbounded subset $Y$ such that all bounded subsets of $Y$ are finite. A bornology $\mathcal{B}$ is antitall iff for every unbounded subset $Y$ of $X$ there is a bornologous function on $X$ such that $f$ is unbounded on $Y$.

By Proposition 1 in [12], each bornology is the intersection of tall and antitall bornologies. 


\section{Discrete Balleans}

A ballean $X$ is discrete if $X$ is not bounded and for any $E \in \mathcal{E}_{X}$ there exists a bounded set $B \subset X$ such that $E[x]=\{x\}$ for all $x \in X \backslash B$. More information on discrete balleans can be found in ([5], Chapter 3).

Example 2. Let $X$ be a discrete ballean with bornology that coincides with the family $[X] \leq \omega$ of at most countable sets on an uncountable set $X$. The ballean $X$ is mu-bounded, but each function $f: X \rightarrow \mathbb{R}$ is slowly oscillating and hence eventually macro-uniform. So, $X$ is not emu-bounded and not so-bounded.

Since each macro-uniform function is eventually macro-uniform, each emu-bounded ballean is mu-bounded. Example 2 shows that the converse implication does not hold, even for discrete balleans.

Proposition 1. For a ballean $X$, the following statements are equivalent:

1. Every function $f: X \rightarrow \mathbb{R}$ is macro-uniform.

2. $X$ is discrete and $\mathcal{B}_{X}=[X]^{<\omega}$.

Proof. (2) $\Rightarrow(1)$ is evident. To verify $(1) \Rightarrow(2)$, assume that each real-valued function on $X$ is macro-uniform. In this case, $\mathcal{B}_{X}=[X]^{<\omega}$. To derive a contradiction, assume that $X$ is not discrete. Then there exists $E \in \mathcal{E}_{X}$ and a sequence $\left(x_{n}\right)_{n \in \omega}$ in $X$ such that the subsets $\left\{E\left[x_{n}\right]: n \in \omega\right\}$ are pairwise disjoint and $\left|E\left[x_{n}\right]\right|>1$ for all $n \in \omega$. Then the function $f: X \rightarrow \mathbb{R}$ defined by $f\left(x_{n}\right)=n$ and $f(x)=0$ for $x \notin\left\{x_{n}: n \in \omega\right\}$ is not macro-uniform, which contradicts our assumption.

The following characterization can be easily derived from the definitions.

Proposition 2. For a discrete ballean $X$, the following conditions are equivalent:

1. $X$ is b-bounded.

2. $X$ is mu-bounded.

3. The bornology of $X$ is tall.

A characterization of emu-bounded discrete balleans is more complicated and involves countably complete ultrafilters.

A filter $\mathcal{F}$ on a set $X$ is called countably-complete if for any countable subfamily $\mathcal{C} \subset \mathcal{F}$ the intersection $\cap \mathcal{C}$ belongs to the filter $\mathcal{F}$.

A cardinal $\kappa$ is measurable if there exists a countably complete free ultrafilter $\mathcal{U}$ on $\kappa$. A measurable cardinal, if it exists, is not smaller than the first strongly inaccessible cardinal, see ([13], Chapter 20). This implies that the existence of measurable cardinals cannot be proved in Zermelo-Fraenkel set theory (ZFC).

Proposition 3. For a ballean $X$, the following statements are equivalent:

1. Every function $f: X \rightarrow \mathbb{R}$ is slowly oscillating.

2. Every function $f: X \rightarrow\{0,1\}$ is slowly oscillating.

3. $\mathrm{X}$ is discrete.

If any unbounded subset of $X$ contains an unbounded set of non-measurable cardinality, then conditions (1)-(3) are equivalent to: 
4. Every function $f: X \rightarrow \mathbb{R}$ is boundedly oscillating.

5. Every function $f: X \rightarrow \mathbb{R}$ is eventually macro-uniform.

In particular, conditions (1)-(5) are equivalent if no measurable cardinal exists.

Proof. The equivalence of conditions (1)-(3) has been proven in Theorem 3.3.1 of [5], and the implications $(1) \Rightarrow(4) \Rightarrow(5)$ are trivial. Now, assuming that any unbounded subset of $X$ contains an unbounded set of non-measurable cardinality, we shall prove that $(5) \Rightarrow(3)$ (which is equivalent to $\neg(3) \Rightarrow \neg(5)$ ). Assume that $X$ is not discrete. Then there exists an entourage $E \in \mathcal{E}_{X}$ such that the set $L=\{x \in X: E[x] \neq\{x\}\}$ is unbounded. Using Zorn's Lemma, find a maximal subset $M \subset L$ such that $E[x] \cap E[y]=\varnothing$ for any distinct elements $x, y \in M$. The maximality of $M$ ensures that $L \subset E^{-1}[E[M]]$, which implies that the set $M$ is unbounded. By our assumption, the unbounded set $M$ contains an unbounded subset $N \subset M$ of non-measurable cardinality $|N|$. Using the Zorn's Lemma, extend the free filter $\left\{N \backslash B: B \in \mathcal{B}_{X}\right\}$ to any (free) ultrafilter $\mathcal{U}$. Since $N \in \mathcal{U}$ and the cardinal $|N|$ are not measurable, the ultrafilter $\mathcal{U}$ is not countably-complete, so there exists a decreasing sequence of subsets $\left\{U_{n}\right\}_{n \in \omega} \subset \mathcal{U}$ of $N$ such that $\bigcap_{n \in \omega} U_{n} \notin \mathcal{U}$. Consider the function $f: X \rightarrow \mathbb{R}$ assigning to each $x \in X$ the number:

$$
f(x)= \begin{cases}n & \text { if } x \in U_{n} \backslash U_{n+1} \text { for some } n \in \omega ; \\ 0 & \text { otherwise. }\end{cases}
$$

Assuming that $f$ is eventually macro-uniform, we could find a bounded set $B \subset X$ such that $C:=\sup _{x \in X \backslash B} \operatorname{diam} f(E[x])<\infty$. Choose any number $n \in \omega$ with $n>C$. It follows from $U_{n} \backslash B=U_{n} \cap(X \backslash B) \in \mathcal{U}$ and $\bigcap_{m \in \omega} U_{m} \notin \mathcal{U}$ that $U_{n} \backslash B \not \subset \bigcap_{m \in \omega} U_{m}$ and hence $U_{n} \backslash B \not \subset U_{m}$ for some $m \in \omega$. So, we can find a point $x \in U_{n} \backslash B$ such that $x \notin U_{m}$. It follows that $x \in U_{k} \backslash U_{k+1}$ for some $k \in[n, m)$ and hence diam $f(E[x]) \geq \operatorname{diam}\{f(x), 0\}=k \geq n>C$, which contradicts the definition of $C$. This contradiction shows that the function $f$ is not eventually macro-uniform, which means that condition (5) does not hold.

Example 3. Let $X$ be a set of measurable cardinality $|X|$. We define a coarse structure $\mathcal{E}$ on $X$ such that $(X, \mathcal{E})$ is not discrete but each function $f: X \rightarrow \mathbb{R}$ is eventually macro-uniform. Write $X$ as the union $X=Y \cup Z$ of two disjoint sets of the same cardinality and fix any bijection $h: Y \rightarrow Z$. Let $\Gamma:=\{(x, h(x)): x \in Y\}$ be the graph of the bijection $h$.

By the measurability of the cardinal $|X|=|Y|$, there exists a countably complete ultrafilter $p$ on $Y$. For any set $P \in p$, consider the subset:

$$
B_{P}:=(Y \backslash P) \cup h(Y \backslash P)
$$

of $X$ and the entourage:

$$
E_{P}:=\left(B_{P} \times B_{P}\right) \cup \Delta_{X} \cup \Gamma \cup \Gamma^{-1}
$$

on $X$. Let $\mathcal{E}$ be the coarse structure on $X$, generated by the base $\left\{E_{P}: P \in p\right\}$. It is easy to check that the bornology of the coarse space $(X, \mathcal{E})$ is generated by the base $\left\{B_{P}: P \in p\right\}$. Using this fact and looking at the definition of the basic entourages $E_{P}$, we can conclude that the ballean $\left(X, \mathcal{E}_{X}\right)$ is not discrete.

Now we check that each function $f: X \rightarrow \mathbb{R}$ is eventually macro-uniform. Given any set $P \in p$, we should find a bounded set $B \in \mathcal{B}_{X}$ such that $\sup _{x \in X \backslash B} \operatorname{diam} f\left(E_{P}[x]\right)<\infty$. Observe that for any $x \in P=Y \backslash B_{P}$ we get $E_{P}[x]=\{x, h(x)\}=E_{P}[h(x)]$. This implies that diam $f\left(E_{P}[x]\right)<\infty$ and hence $P=\bigcup_{n \in \omega} P_{n}$ where $P_{n}=\left\{x \in P: \operatorname{diam} f\left(E_{P}[x]\right) \leq n\right\}$ for $n \in \omega$. Since the 
ultrafilter $p$ is countably complete, there exists $n \in \omega$ such that $P_{n} \in p$. Then for the bounded set $B=B_{P_{n}}$ and any $x \in X \backslash B$ we get $\operatorname{diam} f\left(E_{P}[x]\right) \leq n$. Indeed, if $x \in Y$, then $x \in Y \backslash$ $B_{P_{n}}=P_{n}$ and hence $\operatorname{diam} f\left(E_{P}[x]\right)=\operatorname{diam}\{x, h(x)\} \leq n$ by the definition of $P_{n}$. If $x \in Z$, then $x \in Z \backslash B_{P_{n}}=h\left(P_{n}\right)$ and then $h^{-1}(x) \in P_{n}$ and $E_{P}[x]=\left\{x, h^{-1}(x)\right\}=E_{P}\left[h^{-1}(x)\right]$ and $\operatorname{diam} f\left(E_{P}[x]\right)=\operatorname{diam} f\left(E_{P}\left[h^{-1}(x)\right]\right) \leq n$.

Given a ballean $X$, we denote by $X^{\sharp}$ the set of all ultrafilters $p$ on $X$ such that each member of $p$ is unbounded in $X$. It is easy to see that the set $X^{\sharp}$ is closed in the Stone-Čech extension $\beta X$ of $X$ endowed with the discrete topology.

Proposition 4. For a discrete ballean $X$, the following statements are equivalent:

1. $\mathrm{X}$ is emu-bounded.

2. $X$ is bo-bounded.

3. $X$ is so-bounded.

4. $\quad X^{\sharp}$ is finite and each ultrafilter $p \in X^{\sharp}$ is countably complete.

5. each ultrafilter $p \in X^{\sharp}$ is countably complete.

Proof. The implication (3) $\Rightarrow(4)$ is proven in Theorem 3.3.2 of [5], and (1) $\Rightarrow(2) \Rightarrow$ (3) are evident.

$(4) \Rightarrow(1)$ Assume that $X^{\sharp}$ is finite and each ultrafilter $p \in X^{\sharp}$ is countably complete. To show that $X$ is emu-bounded, take any eventually macro-uniform function $f: X \rightarrow \mathbb{R}$. Let $X^{\sharp}=\left\{p_{1}, \ldots, p_{k}\right\}$ and observe that $\bigcap_{i=1}^{k} p_{k}=\left\{X \backslash B: B \in \mathcal{B}_{X}\right\}$ where $\mathcal{B}_{X}$ is the bornology of the ballean $X$. For every $n \in \omega$ consider the set $X_{n}=\{x \in X:|f(x)| \leq n\}$ and observe that $X=\bigcup_{n \in \omega} X_{n}$. For every $i \leq k$, the countable completeness of the ultrafilter $p_{i} \ni \bigcup_{n \in \omega} X_{n}$ yields a number $n_{i} \in \omega$ such that $X_{n_{i}} \in p_{i}$. Then for the number $n=\max _{i \leq k} n_{i}$, we obtain $X_{n} \in \bigcap_{i=1}^{k} p_{i}=\left\{X \backslash B: B \in \mathcal{B}_{X}\right\}$. Therefore, the complement $B:=X \backslash X_{n}$ is bounded and $f(X \backslash B)=f\left(X_{n}\right) \subset[-n, n]$ is bounded in the real line.

The implication $(4) \Rightarrow(5)$ is trivial. To prove that $(5) \Rightarrow(4)$, assume that the set $X^{\sharp}$ is infinite and hence contains a sequence $\left(p_{n}\right)_{n \in \omega}$ of pairwise distinct ultrafilters. Since $X^{\sharp}$ is a closed subspace of the compact Hausdorff space $\beta X$, we can replace $\left(p_{n}\right)_{n \in \omega}$ by a suitable subsequence and assume that the subspace $\left\{p_{n}\right\}_{n \in \omega}$ is discrete in $X^{\sharp}$. Fix any free ultrafilter $\mathcal{U}$ on the set $\omega$ and consider the ultrafilter $p=\lim _{n \rightarrow \mathcal{U}} p_{n}$, consisting of the sets $\bigcup_{n \in \mathcal{U}} P_{n}$ where $U \in \mathcal{U}$ and $P_{n} \in p_{n}$ for all $n \in U$. Since the set $X^{\sharp}$ is closed in $\beta X$, the ultrafilter $p$ belongs to the set $X^{\sharp}$, being an accumulation point of the set $\left\{p_{n}: n \in \omega\right\}$ in $\beta X$. We claim that the ultrafilter $p$ is not countably complete. Using the discreteness of the subspace $\left\{p_{n}\right\}_{n \in \omega}$ in $X^{\sharp}$, in each ultrafilter $p_{n}$ we can choose a set $P_{n}$ so that the family $\left(P_{n}\right)_{n \in \omega}$ is disjoint. Now observe that for every $n \in \omega$ the set $Q_{n}=\bigcup_{m \geq n} P_{m}$ belongs to the ultrafilter $p$ but $\bigcap_{n \in \omega} Q_{n}=\varnothing$, witnessing that $p$ is not countably complete.

A discrete ballean $X$ is called ultradiscrete if the family $\left\{X \backslash B: B \in \mathcal{B}_{X}\right\}$ is an ultrafilter on $X$. Proposition 4 implies the following characterization.

Corollary 1. For an ultradiscrete ballean $X$, the following statements are equivalent:

1. $\mathrm{X}$ is emu-bounded.

2. $X$ is bo-bounded.

3. $X$ is so-bounded.

4. The bornology $\mathcal{B}_{X}$ of $X$ is $\sigma$-additive. 


\section{Products}

In this section, we discuss the problem of preservation of various notions of boundedness by products of balleans.

Theorem 1. The product $X \times Y$ of two mu-bounded balleans $X, Y$ is mu-bounded.

Proof. Let $f: X \times Y \rightarrow \mathbb{R}$ be a mu-function. For each $x \in X$, the function $f_{x}: Y \rightarrow \mathbb{R}$, defined as $f_{x}(y)=f(x, y)$, is a mu-function, so $f_{x}$ is bounded. It is easy to check that the function $s: X \rightarrow \mathbb{R}$, $s: x \mapsto \sup \left\{\left|f_{x}(y)\right|: y \in Y\right\}$ is macro-uniform and hence bounded (as $X$ is mu-bounded). Then the function $f$ is bounded, as well.

Theorem 2. Let $X, Y$ be balleans and $Y$ is either bounded or locally finite. If $X, Y$ are either emu-bounded or bo-bounded or so-bounded, then the product $X \times Y$ has the same property.

Proof. We proved only the third statement. Assume that the balleans $X, Y$ are so-bounded. Take any so-function $f: X \times Y \rightarrow \mathbb{R}$. Assume that $Y$ is bounded, fix any $y_{0} \in Y$ and consider the so-function $f_{y_{0}}: X \rightarrow \mathbb{R}, f_{y_{0}}: x \mapsto f\left(x, y_{0}\right)$. Since $X$ is so-bounded, there exists a bounded set $B \subset X$ such that $f_{y_{0}}$ is bounded on $X \backslash B$. Since $Y$ is bounded and $f$ is slowly oscillating, we can replace $B$ by a larger bounded set, and assume that the number $c:=\sup _{x \in X \backslash B} \operatorname{diam} f(\{x\} \times Y)$ is finite. Then $f$ is bounded on $(X \times Y) \backslash(B \times Y)$.

Now assume that $Y$ is locally finite. In this case, for every $x \in X$ the so-function $f_{x}: Y \rightarrow \mathbb{R}$, $f_{x}: y \mapsto f(x, y)$, is bounded. Then the function $s: X \rightarrow \mathbb{R}, s: x \mapsto \sup _{y \in Y}\left|f_{x}(y)\right|$, is well-defined and slowly oscillating. Since $X$ is so-bounded, there is $B \in \mathcal{B}_{X}$ such $s$ is bounded on $X \backslash B$. Then $f$ is bounded on $(X \times Y) \backslash(B \times Y)$. By first case, there is a bounded subset $C$ of $Y$ such $f$ is bounded on $(B \times Y) \backslash(B \times C)$.

Question 1. Is the product of any two emu-bounded balleans emu-bounded?

Question 2. Is the product of any two bo-bounded balleans bo-bounded?

Question 3. Is the product of any two so-bounded balleans so-bounded?

For more results on products of balleans, see [14]. Now, we give some partial answers to Questions 1-3.

For any bornology $\mathcal{B}$ on a set $X \notin \mathcal{B}$, consider the cardinal characteristics:

$$
\begin{aligned}
\operatorname{add}(\mathcal{B}) & =\min \{|\mathcal{A}|: \mathcal{A} \subset \mathcal{B}, \cup \mathcal{A} \notin \mathcal{B}\} \\
\operatorname{non}(\mathcal{B}) & =\min \{|A|: A \subset X, \quad A \notin \mathcal{B}\} \\
\operatorname{cov}(\mathcal{B}) & =\min \{|\mathcal{A}|: \mathcal{A} \subset \mathcal{B}, \cup \mathcal{A}=X\}, \\
\operatorname{cof}(\mathcal{B}) & =\min \{|\mathcal{A}|: \mathcal{A} \subset \mathcal{B}, \quad \forall B \in \mathcal{B} \exists A \in \mathcal{A}(B \subset A)\}
\end{aligned}
$$

It is known (and easy to see) that:

$$
\operatorname{add}(\mathcal{B}) \leq \min \{\operatorname{cov}(\mathcal{B}), \operatorname{non}(\mathcal{B})\} \leq \max \{\operatorname{cov}(\mathcal{B}), \operatorname{non}(\mathcal{B})\} \leq \operatorname{cof}(\mathcal{B}) .
$$

Proposition 5. Let $X, Y$ be two balleans with $\operatorname{cof}\left(\mathcal{B}_{X}\right)<\operatorname{non}\left(\mathcal{B}_{Y}\right)$. If $X$ and $Y$ are emu-bounded (bo-bounded or so-bounded, respectively), then so is the product $X \times Y$. 
Proof. We shall present a proof only for the case of emu-boundedness. The other two cases can be considered by analogy. Assume that the balleans $X, Y$ are emu-bounded. If the ballean $X$ is bounded, then the product $X \times Y$ is emu-bounded by Theorem 2. So we assume that $X$ is unbounded. By the definition of the cardinal $\kappa=\operatorname{cof}\left(\mathcal{B}_{X}\right)$, the bornology $\mathcal{B}_{X}$ of $X$ has a base $\mathcal{B} \subset \mathcal{B}_{X}$ of cardinality $|\mathcal{B}|=\kappa$.

To prove that the product $X \times Y$ is emu-bounded, take any emu-function $f: X \times Y \rightarrow \mathbb{R}$. Fix any point $y_{0} \in Y$. Since the function $f_{y_{0}}: X \rightarrow \mathbb{R}, f_{y_{0}}: x \mapsto f\left(x, y_{0}\right)$, is emu-bounded, for any entourage $E \in \mathcal{E}_{Y}$ there exists a bounded set $B_{E} \in \mathcal{B}$ such that:

$$
c_{E}:=\sup _{x \in X \backslash B_{E}} \operatorname{diam} f\left(\{x\} \times E\left[y_{0}\right]\right)
$$

is finite. We claim that for some $B \in \mathcal{B}$ and $m \in \mathbb{N}$, the union

$$
U_{B, m}:=\bigcup\left\{E\left[y_{0}\right]: E \in \mathcal{E}_{Y}, B_{E}=B, c_{E} \leq m\right\}
$$

equals $Y$. To derive a contradiction, assume that for every $B \in \mathcal{B}$ and $m \in \mathbb{N}$ the union $U_{B, m}$ does not contain some point $y_{B, m} \in Y$. Since $\omega \leq \kappa<\operatorname{non}\left(\mathcal{B}_{Y}\right)$, the set $V=\left\{y_{B, m}: B \in \mathcal{B}, m \in \mathbb{N}\right\}$ is bounded in $Y$ and hence is contained in the ball $E\left[y_{0}\right]$ for some $E \in \mathcal{E}_{Y}$. Then for the set $B=B_{E}$ and any number $m \in \mathbb{N}$ with $m \geq c_{E}$, we get $y_{B, m} \in E\left[y_{0}\right] \subset U_{B, m}$, which contradicts the choice of $y_{B, m}$. This contradiction shows that $U_{B, m}=Y$ for some $B \in \mathcal{B}$ and $m \in \mathbb{N}$.

Then $\operatorname{diam} f(\{x\} \times Y) \leq 2 m$ for each $x \in X \backslash B$. Since $X$ is emu-bounded, there exists $D \in \mathcal{B}_{X}$ such that the number $c:=\sup _{x \in X \backslash D}\left|f\left(x, y_{0}\right)\right|$ is finite. Replacing $D$ by $B \cup D$, we can assume that $B \subset D$. Then $|f(x, y)|<c+2 m$ for all $(x, y) \in(X \backslash D) \times Y$. By Theorem 2, the ballean $D \times Y$ is emu-bounded, which implies that the function $f$ is bounded on the complement $D \times Y \backslash D \times C$ for some bounded set $C \in \mathcal{B}_{Y}$. Now we see that $f$ is bounded on $X \times Y \backslash D \times C$, witnessing that the ballean $X \times Y$ is emu-bounded.

Proposition 6. Let $X, Y$ be two so-bounded balleans. If $\operatorname{cov}\left(\mathcal{B}_{X}\right)<\operatorname{add}\left(\mathcal{B}_{Y}\right)$, then the product $X \times Y$ is so-bounded.

Proof. By the definition of the cardinal $\kappa=\operatorname{cov}\left(\mathcal{B}_{X}\right)$, there exists a subfamily $\left\{B_{\alpha}\right\}_{\alpha \in \kappa} \subset \mathcal{B}_{X}$ with $\bigcup_{\alpha \in \kappa} B_{\alpha} \in X$.

To show that the ballean $X \times Y$ is so-bounded, take any so-function $f: X \times Y \rightarrow \mathbb{R}$. Fix any point $x_{0} \in X$, and for every $\alpha \in \kappa$ find an entourage $E_{\alpha}$ on $X$ such that $B_{\alpha} \subset$ $E_{\alpha}\left[x_{0}\right]$. Then $X=\cup_{\alpha \in \kappa} E_{\alpha}\left[x_{0}\right]$. Since $f$ is slowly oscillating, there exists $C_{\alpha} \in \mathcal{B}_{Y}$ such that $\sup _{y \in Y \backslash C_{\alpha}} \operatorname{diam} f\left(E_{\alpha}\left[x_{0}\right] \times\{y\}\right) \leq 1$. Since $\operatorname{add}\left(\mathcal{B}_{Y}\right)>\operatorname{cov}\left(\mathcal{B}_{X}\right)=\kappa$, the set $C=\bigcup_{\alpha<\kappa} C_{\alpha}$ is bounded in $Y$. If $y \in Y \backslash C$, then $\operatorname{diam} f\left(E_{\alpha}\left[x_{0}\right] \times\{y\}\right) \leq 1$ for every $\alpha \in \kappa$ and hence $\operatorname{diam} f(X \times\{y\}) \leq 2$.

Since $Y$ is so-bounded, there exists a bounded set $D \in \mathcal{B}_{Y}$ such that $\sup _{y \in Y \backslash D}\left|f\left(x_{0}, y\right)\right|<1$. Replacing $D$ by $C \cup D$, we can assume that $C \subset D$. Then $|f(x, y)|<1+\operatorname{diam} f(X \times\{y\}) \leq$ $1+2=3$ for all $x \in X$ and $y \in Y \backslash D$. Therefore, $f$ is bounded on the set $(X \times Y) \backslash(X \times D)$.

By Theorem 2, $X \times D$ is so-bounded, so there exists a bounded set $B \subset X$ such that $f$ is bounded on the set $(X \times D) \backslash(B \times D)$. Summing up, we conclude that $f$ is bounded on the complement of the bounded set $B \times D$.

Proposition 7. Let $X, Y$ be two balleans such that $\operatorname{cov}\left(\mathcal{B}_{X}\right)<\operatorname{add}\left(\mathcal{B}_{Y}\right)$ and the bornology $\mathcal{B}_{X}$ is tall. If $X$ and $Y$ are emu-bounded (respectively, bo-bounded), then so is the product $X \times Y$. 
Proof. We shall provide a detailed proof only for the case of emu-boundedness. The case of bo-boundedness can be considered by analogy. So assume that the balleans $X, Y$ are emu-bounded. By the definition of the cardinal $\kappa=\operatorname{cov}\left(\mathcal{B}_{X}\right)$, there exists a subfamily $\left\{B_{\alpha}\right\}_{\alpha \in \kappa} \subset \mathcal{B}_{X}$ with $\bigcup_{\alpha \in \kappa} B_{\alpha} \in X$.

To show that the ballean $X \times Y$ is emu-bounded, take any emu-function $f: X \times Y \rightarrow \mathbb{R}$. Fix any point $x_{0} \in X$, and for every $\alpha \in \mathcal{\kappa}$ find an entourage $E_{\alpha}$ on $X$ such that $B_{\alpha} \subset E_{\alpha}\left[x_{0}\right]$. Since $f$ is eventually macro-uniform, there exists $C_{\alpha} \in \mathcal{B}_{Y}$ such that:

$$
c_{\alpha}:=\sup _{y \in Y \backslash C_{\alpha}} \operatorname{diam} f\left(E_{\alpha}\left[x_{0}\right] \times\{y\}\right)
$$

is finite. For every $m \in \mathbb{N}$ let $K_{m}=\left\{\alpha \in \kappa: c_{\alpha} \leq m\right\}$. We claim that for some $m \in \mathbb{N}$ the union $U_{m}=\bigcup_{\alpha \in K_{m}} E_{\alpha}\left[x_{0}\right]$ coincides with $X$. In the opposite case, for every $m \in \mathbb{N}$, we can choose a point $x_{m} \in X \backslash U_{m}$. Since the bornology $\mathcal{B}_{X}$ is tall, there exists an increasing number sequence $\left(m_{k}\right)_{k \in \omega}$ such that the set $\left\{x_{m_{k}}\right\}_{k \in \omega}$ is bounded and hence is contained in $B_{\alpha} \subset E_{\alpha}\left[x_{0}\right]$ for some $\alpha \in \kappa$. Then, for any $k \in \omega$ with $m_{k} \geq c_{\alpha}$, we have $x_{m_{k}} \in E_{\alpha}\left[x_{0}\right] \subset U_{m_{k}}$, which contradicts the choice of $x_{m_{k}}$. This contradiction shows that $U_{m}=X$ for some $m \in \mathbb{N}$.

Since $\operatorname{add}\left(\mathcal{B}_{Y}\right)>\operatorname{cov}\left(\mathcal{B}_{X}\right)=\kappa \geq\left|K_{m}\right|$, the set $C=\bigcup_{\alpha \in K_{m}} C_{\alpha}$ is bounded in $Y$. If $y \in Y \backslash C$ then diam $f\left(E_{\alpha}\left[x_{0}\right] \times\{y\}\right) \leq c_{\alpha} \leq m$ for every $\alpha \in K_{m}$ and hence diam $f(X \times\{y\})=\operatorname{diam}\left(U_{m} \times\right.$ $\{y\}) \leq 2 m$.

Since $Y$ is emu-bounded, there exists a bounded set $D \in \mathcal{B}_{Y}$ such that the number

$$
c:=\sup _{y \in Y \backslash D}\left|f\left(x_{0}, y\right)\right|
$$

is finite. Replacing the set $D$ by $C \cup D$, we can assume that $C \subset D$. Then $|f(x, y)|<c+$ $\operatorname{diam} f(X \times\{y\})<c+2 m$ for all $x \in X$ and $y \in Y \backslash D$. Therefore, $f$ is bounded on the set $(X \times Y) \backslash(X \times D)$.

By Theorem 2, the ballean $X \times D$ is emu-bounded, so there exists a bounded set $B \subset X$ such that $f$ is bounded on the set $(X \times D) \backslash(B \times D)$. Summing up, we conclude that $f$ is bounded on $(X \times Y) \backslash(B \times D)$, witnessing that $X \times Y$ is emu-bounded.

\section{Group Balleans}

A bornology $\mathcal{B}$ on a group $G$ is called a group bornology if for any sets $A, B \in \mathcal{B}$ the set $A B^{-1}=\left\{a b^{-1}: a \in A, b \in B\right\}$ belongs to $\mathcal{B}$.

Each group bornology $\mathcal{B}$ on a group $G$ induces the coarse structure $\mathcal{E}_{\mathcal{B}}$, generated by the base $\left\{E_{B}: B \in \mathcal{B}\right\}$ consisting of the entourages $E_{B}=\{(x, y) \in G \times G: y \in\{x\} \cup B x\}$ where $B \in \mathcal{B}$.

It is easy to see that for any infinite cardinal $\kappa$ and any group $G$, the family

$$
[G]^{<\kappa}:=\{B \subset G:|B|<\kappa\}
$$

is a group bornology on $G$. It induces the coarse structure $\mathcal{E}_{[G]}<\kappa$ on $G$. The ballean $\left(G, \mathcal{E}_{[G]^{<\kappa}}\right)$ will be called the $\kappa$-ballean of $G$. The $\omega$-ballean of $G$ is called the finitary ballean of $G$. It should be mentioned that the finitary balleans of finitely generated groups $G$ have been intensively studied in Geometrical Group Theory, see [2, Chapter 4], Chapter 4. For properties of the $\kappa$-balleans of groups, see [15-17].

Theorem 3. For any infinite regular cardinal $\kappa$, the $\kappa$-ballean of any group $G$ of cardinality $|G|=\kappa$ is not so-bounded. 
Proof. Since $\kappa$ is regular, the coarse structure $\mathcal{E}_{[G]^{<\kappa}}$ has a linearly ordered base. By Proposition 3.1 of [18], the $\kappa$-ballean $\left(G, \mathcal{E}_{[G]<\kappa}\right)$ is not so-bounded.

Example 3.3 from [19] shows that the finitary ballean of any infinite free group $F(X)$ is not so-bounded.

Theorem 4. For any infinite cardinal $\kappa$ and set $X$ of cardinality $|X| \geq \kappa$ the $\kappa$-ballean of the free group $G=F(X)$ is not so-bounded.

Proof. We partition $X=\bigcup_{n \in \mathbb{N}} X_{n}$ so that $\left|X_{n}\right|=|X|$ for each $n \in \mathbb{N}$, and define a function $h: X \cup\{e\} \cup X^{-1} \rightarrow \mathbb{R}$ by $h(e)=0$ and $h(x)=h\left(x^{-1}\right)=n$ for any $x \in X_{n}, n \in \mathbb{N}$. Then we define a mapping $f: G \rightarrow \mathbb{R}$, assigning to each word $w \in G=F(X)$ the number $h(a)$ where $a$ is the last letter in the irreducible representation of $w$. It is easy to see that $f$ is unbounded on the complement $G \backslash B$ of any set $B \in[G]^{<\kappa}$.

To show that $f$ is slowly oscillating, we take an arbitrary subset $B \in[G]^{<\kappa}$ and denote by $S$ the subgroup of $G$ generated by all letters which appear in the (irreducible) words from $B$. If $w \in G \backslash S$ and $y \in S$, then $f(y w)=f(w)$, so $\operatorname{diam} f(S w)=0$.

Following [20], we define group $G$ to be $\kappa$-normal if every subset $F \in[G]^{<\kappa}$ is contained in a normal subgroup $H \in[G]^{<\kappa}$ of $G$.

Theorem 5. Let $\kappa$ be a cardinal of uncountable cofinality and $G$ be a group of cardinality $|G|>\kappa$. If the group $G$ is $\kappa^{+}$-normal, then the $\kappa$-ballean of $G$ is emu-bounded.

Proof. Given any emu-function $f: G \rightarrow \mathbb{R}$, we should prove that $f$ is bounded on the complement $G \backslash B$ of some set $B \in[G]^{<\kappa}$. First, we prove two claims.

Claim 1. There exists a normal subgroup $H \subset G$ of cardinality $\kappa$ such that:

$$
\sup _{x \in G \backslash H} \operatorname{diam} f(H x)<\infty .
$$

Proof. Since $G$ is a $\kappa^{+}$-normal group of cardinality $|G|>\kappa$, there exists a normal subgroup $H_{0} \subset G$ of cardinality $\left|H_{0}\right|=\kappa$. By transfinite induction of length $\omega_{1}$, we shall construct an increasing sequence $\left(H_{\alpha}\right)_{\alpha \in \omega_{1}}$ of normal subgroups of cardinality $\kappa$ such that for any ordinal $\alpha<\omega_{1}$ the supremum $\sup _{x \in X \backslash H_{\alpha+1}} \operatorname{diam} f\left(H_{\alpha} x\right)$ is finite.

Assume that, for some non-zero ordinal $\alpha<\omega_{1}$, we have constructed an increasing sequence $\left(H_{\beta}\right)_{\beta<\alpha}$ of normal subgroups of cardinality $\kappa$ in $G$ such that for any ordinal $\beta$ with $\beta+1<\alpha$ the supremum $\sup _{x \in X \backslash H_{\beta+1}} \operatorname{diam} f\left(H_{\beta} x\right)$ is finite. If $\alpha$ is a limit ordinal, put $H_{\alpha}=\bigcup_{\beta<\alpha} H_{\beta}$.

If $\alpha=\beta+1$ is a successor ordinal, then write the group $H_{\beta}$ as the union $H_{\beta}=\bigcup_{i \in \mathrm{cf}(\kappa)} \Gamma_{i}$ of an increasing sequence $\left(\Gamma_{i}\right)_{i \in \mathrm{cf}(\kappa)}$ of sets of cardinality $\left|\Gamma_{i}\right|<\kappa$. Since $f$ is eventually macro-uniform, for every $i \in \operatorname{cf}(\kappa)$ there exists a set $B_{i} \in[G]^{<\kappa}$ such that $c_{i}:=\sup _{x \in G \backslash B_{i}} \operatorname{diam} f\left(\Gamma_{i} x\right)$ is finite. Since $G$ is $\kappa^{+}$-normal, the set $H_{\beta} \cup \bigcup_{i \in \mathrm{cf}(\kappa)} B_{i}$ is contained in a normal subgroup $H_{\alpha}$ of cardinality $\left|H_{\alpha}\right|=\kappa$. Since the cardinal $\operatorname{cf}(\kappa)$ is uncountable and regular, there exists $c \in \mathbb{N}$ such that the set $\Omega=\left\{i \in \operatorname{cf}(\kappa): c_{i} \leq c\right\}$ is unbounded in $\operatorname{cf}(\kappa)$. Then $H_{\beta}=\bigcup_{i \in \Omega} \Gamma_{i}$ and for any $x \in X \backslash H_{\alpha}=X \backslash H_{\beta+1}$ :

$$
\operatorname{diam} f\left(H_{\beta} x\right)=\sup _{i \in \Omega} \operatorname{diam} f\left(\Gamma_{i} x\right) \leq \sup _{i \in \Omega} c_{i} \leq c .
$$


Claim 2. The function $f$ is bounded on the set $G \backslash H$.

Proof. To derive a contradiction, assume that $f$ is unbounded on $G \backslash H$ and choose a sequence $\left(g_{n}\right)_{n \in \omega}$ in $G \backslash H$ such that the set $\left\{f\left(g_{n}\right): n \in \omega\right\}$ is unbounded. We put $K=\left\{g_{n} g_{0}^{-1}: n \in \omega\right\}$ and choose $B \in[G]^{<\kappa}$ such that the number

$$
C:=\sup _{x \in X \backslash B} \operatorname{diam} f(K x)
$$

is finite. By Claim 1, the supremum

$$
c:=\sup _{x \in G \backslash H} \operatorname{diam} f(H x)
$$

is finite. Since $|H|=\kappa>|B|=\left|g_{0}^{-1} B\right|$, there exists $x \in H \backslash g_{0}^{-1} B$. Then $g_{0} x \notin B$ and $\left|f\left(g_{n} g_{0}^{-1}\left(g_{0} x\right)\right)-f\left(g_{0} x\right)\right| \leq \operatorname{diam} f(K x) \leq C$ for each $n>0$. Since $H$ is normal, $g_{n} x \in g_{n} H=$ $H g_{n}$, and then

$$
\begin{aligned}
\left|f\left(g_{n}\right)\right| & =\left|f\left(g_{n}\right)-f\left(g_{n} x\right)+f\left(g_{n} x\right)-f\left(g_{0} x\right)+f\left(g_{0} x\right)\right| \leq \\
& \leq\left|f\left(g_{n}\right)-f\left(g_{n} x\right)\right|+\left|f\left(g_{n} x\right)-f\left(g_{0} x\right)\right|+\left|f\left(g_{0} x\right)\right|< \\
& <\operatorname{diam} f\left(H g_{n}\right)+C+\left|f\left(g_{0} x\right)\right|<c+C+\left|f\left(g_{0} x\right)\right|
\end{aligned}
$$

for every $n$, which contradicts the choice of $\left(g_{n}\right)_{n \in \omega}$. This contradiction shows that $f$ is bounded on $G \backslash H$.

Fix any element $g \in G \backslash H$ and observe that $g H \subset G \backslash H$, and hence the set $f(g H) \subset f(G \backslash H)$ is bounded in the real line (according to Claim 2). Consequently, the supremum

$$
c:=\sup _{x \in G \backslash H}|f(x)|
$$

is finite.

Since $f$ is eventually macro-uniform, there exists a set $B \in[G]^{<\kappa}$ such that the number

$$
c^{\prime}:=\sup _{x \in G \backslash B}|f(g x)-f(x)|
$$

is finite. Now take any $x \in H \backslash B$ and observe that

$$
|f(x)| \leq|f(g x)-f(x)|+|f(g x)| \leq c^{\prime}+c
$$

witnessing that $f$ is bounded on $H \backslash B$ and, consequently, bounded on $G \backslash B=(G \backslash H) \cup(H \backslash B)$.

A subset $A$ of a group $G$ is defined to be $\kappa$-large if $G=F A$ for some $F \in[G]^{<\kappa}$. In the proof of Theorem 7, we shall need the following result, proven in [20].

Theorem 6 (Protasov, Slobodianiuk). Let $\kappa$ be a singular cardinal and let $G$ be a $\kappa$-normal group of cardinality $\kappa$. For every finite partition of $G$, at least one cell of the partition is $\kappa$-large.

Theorem 7. Let $\kappa$ be a singular cardinal and let $G$ be a group of cardinality $|G|=\kappa$. If the group $G$ is $\kappa$-normal, then the $\kappa$-ballean of $G$ is emu-bounded. 
Proof. Assuming that the $\kappa$-ballean of $G$ is not emu-bounded, we can find an emu-function $f: G \rightarrow \mathbb{N}$ such that $f(G \backslash B)$ is unbounded for every $|B| \in[G]^{<\kappa}$. This property of $f$ implies that for any $m \in \mathbb{N}$ the preimage $f^{-1}([m, \infty))$ has cardinality $\kappa$.

Claim 3. There exists a number sequence $\left(n_{i}\right)_{i \in \omega}$ such that $n_{0}=0, n_{i+1}-n_{i}>i$ for all $i \in \omega$ and for every $m \in \omega$ and $r \in\{0,1,2,3\}$ the set

$$
X_{m, r}:=\bigcup_{k=m}^{\infty} f^{-1}\left(\left[n_{4 k+r}, n_{4 k+r+1}\right)\right)
$$

has cardinality $\left|X_{m, r}\right|=\kappa$.

Proof. Separately, we shall consider two cases.

1. The set $W=\left\{n \in \omega:\left|f^{-1}([n, n+1))\right|=\kappa\right\}$ is infinite. In this case let $\left\{n_{i}\right\}_{i \in \omega} \subset \omega$ be any increasing number sequence such that $n_{0}=0, n_{i+1}-n_{i}>i$ and $\left[n_{i+1}, n_{i}\right) \cap W \neq \varnothing$ for all $i \in \omega$. It is clear that the sequence $\left(n_{i}\right)_{i \in \omega}$ has the required property.

2. The set $W$ is finite. Put $n_{0}=0$ and find a number $n_{1} \in \mathbb{N}$ with $W \subset\left[n_{0}, n_{1}\right)$. The definition of $W$ implies that for any $m>n_{1}$ the preimage $f^{-1}\left(\left[n_{1}, m\right)\right)$ has cardinality $<\kappa$. Taking into account that $f^{-1}\left(\left[n_{1}, \infty\right)\right)=\bigcup_{m>n_{1}} f^{-1}\left(\left[n_{1}, m\right)\right)$ has cardinality $\kappa$, we conclude that the cardinal $\kappa$ has countable cofinality. So, we can find a strictly increasing sequence of cardinals $\left(\kappa_{i}\right)_{i \in \omega}$ with $\sup _{i \in \omega} \kappa_{i}=\kappa$. Since $f^{-1}\left(\left[n_{1}, \infty\right)\right)=\bigcup_{n>m} f^{-1}\left(\left[n_{1}, m\right)\right)$ has cardinality $\kappa$, there exists a number $n_{2}>n_{1}+1$ such that $\left|f^{-1}\left(\left[n_{1}, n_{2}\right)\right)\right| \geq \kappa_{1}$. Proceeding by induction, for every $i \in \mathbb{N}$ we can find a number $n_{i+1}>n_{i}+i$ such that $\left|f^{-1}\left(\left[n_{i}, n_{i+1}\right)\right)\right|>\kappa_{i}$. It is easy to see that the sequence $\left(n_{i}\right)_{i \in \omega}$ has the required property.

Since $G=\bigcup_{r=0}^{3} X_{0, r}$, we can apply Theorem 6 and find a number $r \in\{0,1,2,3\}$ such that the set $X_{0, r}$ is $\kappa$-large in $G$ and hence $G=F X_{0, r}$ for some set $F \in[G]^{<\kappa}$ containing the unit $e$ of $G$.

Since the function $f$ is eventually macro-uniform, there exists a set $B \in[G]^{<\kappa}$ such that $\sup _{x \in G \backslash B} \operatorname{diam} f(F x)<m$ for some number $m \in \mathbb{N}$. Let $q \in\{0,1,2,3\}$ be a unique number with $|q-r|=2$. Since $\left|X_{m, q}\right|=\kappa>|F B|$, there exists an element $y \in X_{m, q} \backslash F B$. Taking into account that $y \in G \backslash F B=F X_{0, r} \backslash F B=F\left(X_{0, r} \backslash B\right)$, we see that $y \in F x$ for some $x \in X_{0, r} \backslash B$. The choice of $B$ ensures that $\operatorname{diam} f(F x) \leq m$ and hence $|f(y)-f(x)| \leq \operatorname{diam} f(F x) \leq m$. It follows from $x \in X_{0, r}=\bigcup_{k=0}^{\infty} f^{-1}\left(\left[n_{4 k+r}, n_{4 k+r+1}\right)\right)$ that $f(x) \in\left[n_{4 i+r}, n_{4 i+r+1}\right)$ for some $i \in \omega$. On the other hand, $y \in X_{m, q}=\bigcup_{k=m}^{\infty} f^{-1}\left(\left[n_{4 k+q}, n_{4 k+q+1}\right)\right)$ yields a number $j \geq m$ such that $f(y) \in\left[n_{4 j+q}, n_{4 j+q+1}\right)$. Now, we arrive at a contradiction with $|f(y)-f(x)| \leq m$, considering the following four cases.

$$
\begin{aligned}
& \text { If } i<j \text {, then } 4 i+r+1 \leq 4(j-1)+1+r \leq 4 j-3+q+|r-q|=4 j+q-1 \text { and hence } \\
& \quad f(y) \geq n_{4 j+q}>n_{4 j+q-1}+(4 j+q-1) \geq n_{4 i+r+1}+(4 m+q-1)>f(x)+m . \\
& \text { If } j<i \text {, then } 4 j+q+1 \leq 4(i-1)+1+q \leq 4 i-3+r+|r-q|=4 i+r-1 \text { and hence } \\
& \qquad f(x) \geq n_{4 i+r}>n_{4 i+r-1}+(4 i+r-1) \geq n_{4 j+q+1}+(4 j+r+3)>f(y)+m . \\
& \text { If } i=j \text { and } r<q \text {, then } \\
& \quad f(y) \geq n_{4 j+q}>n_{4 j+q-1}+(4 j+q-1)=n_{4 j+r+1}+(4 j+q-1)>f(x)+m .
\end{aligned}
$$


If $i=j$ and $q<r$, then

$$
f(x) \geq n_{4 i+r}>n_{4 i+r-1}+(4 i+r-1)=n_{4 j+q+1}+(4 j+r-1)>f(y)+m .
$$

Theorem 8. Let $G$ be a group. If the $\kappa$-ballean of each subgroup $H$ of $G$ with $|H|=\kappa$ is emu-bounded, then the $\kappa$-ballean of $G$ is emu-bounded.

Proof. Assuming that the $\kappa$-ballean of $G$ is not emu-bounded, we can find an emu-function $f: G \rightarrow \mathbb{N}$, which is unbounded on each subset $G \backslash B,|B|<\kappa$. This property of $f$ implies that for every $m \in \mathbb{N}$ the set $f^{-1}\left([m, \infty)\right.$ has cardinality $\geq \kappa$ and hence contains a subset $X_{m}$ of cardinality $\left|X_{m}\right|=\kappa$. Then the set $\bigcup_{m \in \omega} X_{m}$ generates a subgroup $H \subset G$ of cardinality $|H|=\kappa$ such that $f(H \backslash B)$ is unbounded for any $B \in[H]^{<\kappa}$. Now we see that the restriction $f \nmid H$ witnesses that $H$ is not emu-bounded.

Remark 1. By Theorems 3, 4, 5, 7 and 8, for an uncountable cardinal $\kappa$, the $\kappa$-ballean of an Abelian group $G$ is emu-bounded iff either $\kappa$ is singular or $\kappa$ is regular and $|G|>\kappa$. In this case, the emu-boundedness is equivalent to the so-boundedness. If $\kappa$ is regular and $G$ is an Abelian group of cardinality $|G|>\kappa$, then the $\kappa$-ballean of $G$ is emu-bounded but the $\kappa$-ballean of every subgroup $H,|H|=\kappa$ is not emu-bounded.

Remark 2. By Theorems 3 and 5 , the $\omega_{1}$-ballean of the group $\mathbb{R}$ is emu-bounded iff $\mathrm{CH}$ holds. On the other hand, by Theorem 4, the $\omega_{1}$-ballean of the free group $F(\mathbb{R})$ is not emu-bounded in ZFC.

Question 4. Is the $\omega_{1}$-ballean of the permutation group $S_{\omega}$ emu-bounded in ZFC?

By analogy with Theorem 3.1 of [19], the following theorem can be proven.

Theorem 9. If $G$ is an uncountable $\omega_{1}$-normal group, then the finitary ballean of $G$ is bo-bounded and so-bounded, and every so-function on $G$ is constant at infinity.

Question 5. Assume that the finitary ballean of a group $G$ is so-bounded. Is every so-function on $G$ constant at infinity?

Question 6. Is the so-boundedness of the finitary ballean of a group equivalent to its bo-boundedness?

Finally, we shall discuss the mu-boundedness of finitary balleans on groups. Since the fintary ballean of any group is locally finite, each emu-function is a mu-function. Consequently, the finitary ballean of any group is mu-bounded iff it is emu-bounded.

Definition 1. A group $G$ is defined to be:

- $\quad n$-Shelah for some $n \in \mathbb{N}$ if for each subset $A \subset G$ of cardinality $|A|=|G|$ we have $A^{n}=G$;

- Shelah if it is $n$-Shelah for some $n \in \mathbb{N}$;

- almost Shelah if for each subset $A \subset G$ of cardinality $|A|=|G|$ there exists $n \in \mathbb{N}$ such that $A^{n}=G$;

- Jónsson if each subsemigroup $A \subset G$ of cardinality $|A|=|G|$ coincides with $G$;

- Kurosh if each subgroup $A \subset G$ of cardinality $|A|=|G|$ coincides with $G$; 
- Bergman if $G$ cannot be written as the union a strictly increasing sequence $\left(X_{n}\right)_{n \in \omega}$ of subsets such that $X_{n}=X_{n}^{-1}$ and $X_{n}^{2} \subset X_{n+1}$ for all $n \in \omega$.

For any group $G$, the following implications hold:

$$
\text { finite } \Longleftrightarrow \text { 1-Shelah } \Longrightarrow \text { Shelah } \Longrightarrow \text { almost Shelah } \Longrightarrow \text { Jónsson } \Longrightarrow \text { Kurosh. }
$$

In [8], Shelah constructed a ZFC-example of an uncountable Jónsson group and a CH-example of an infinite 6640-Shelah group. More precisely, for every cardinal $\kappa$ with $\kappa^{+}=2^{\kappa}$, there exists a 6640-Shelah group of cardinality $\kappa^{+}=2^{\kappa}$. In [7], Bergman proved that the permutation group $S_{X}$ of any set $X$ is Bergman. Later it was shown [21-23] that many automorphism groups (of sufficiently homogeneous structures) are Bergman.

Theorem 10. For a group $G$ the following conditions are equivalent:

1. The finitary ballean of $G$ is mu-bounded.

2. The finitary ballean of $G$ is emu-bounded.

3. The group $G$ is Bergman.

The conditions (1)-(3) follow from:

4. $G$ is almost Shelah and $\operatorname{cf}(|G|)>\omega$.

Proof. The equivalence $(1) \Leftrightarrow(2)$ follows from the local finiteness of the finitary ballean on $G$.

$(1) \Rightarrow(3)$. Assume that the group $G$ fails to be Bergman. Then $G=\bigcup_{n \in \omega} X_{n}$ for some strictly increasing sequence of subsets of $G$ such that $X_{n}=X_{n}^{-1}$ and $X_{n}^{2} \subset X_{n+1}$ for all $n \in \omega$. We lose no generality assuming that $X_{0}=\varnothing$.

We claim that the function $\varphi: G \rightarrow \mathbb{N} \subset \mathbb{R}$ assigning to each $x \in X$ the unique number $n \in \mathbb{N}$ such that $x \in X_{n} \backslash X_{n-1}$ is macro-uniform. It follows from $X_{n}^{2} \subset X_{n+1}$ that

$$
\varphi(g x) \leq 1+\max \{\varphi(g), \varphi(x)\} \leq \varphi(x)+(1+\varphi(g))
$$

for every $g, x$. Then $\varphi(x)=\varphi\left(g^{-1} g x\right) \leq \varphi(g x)+\left(1+\varphi\left(g^{-1}\right)\right)$ and hence $\varphi(g x) \geq \varphi(x)-(1+$ $\varphi\left(g^{-1}\right)$. Therefore,

$$
\varphi(x)-\left(1+\varphi\left(g^{-1}\right)\right) \leq \varphi(g x) \leq \varphi(x)+(1+\varphi(g)),
$$

which implies that the map $\varphi$ is macro-uniform. Since the sequence $\left(X_{n}\right)_{n \in \omega}$ is strictly increasing, the map $\varphi$ is unbounded, so the finitary ballean of $G$ is not $m u$-bounded.

(3) $\Rightarrow$ (1) Assume that $G$ admits an unbounded macro-uniform function $\varphi: G \rightarrow \mathbb{R}$. We lose no generality assuming that $\varphi(e)=0$ where $e$ is the unit of the group $G$. Then for every finite set $F \subset G$ there exists a number $n_{F} \in \mathbb{N}$ such that $\varphi(F x) \subset\left[-n_{F}, n_{F}\right]+\varphi(x)$ for every $x \in G$. For every $n \in \mathbb{N}$ let $X_{n}=\left\{g \in G: n_{\left\{g, g^{-1}\right\}} \leq n\right\}$ and observe that $\left(X_{n}\right)_{n \in \omega}$ is an increasing sequence such that $X_{n}=X_{n}^{-1}$ and $\bigcup_{n \in \omega} X_{n}=G$. For every $n \in \omega$ let $Y_{n}=X_{n}^{2^{n}}$ and observe that $Y_{n}=Y_{n}^{-1}$ and $Y_{n} \cdot Y_{n}=X_{n}^{2^{n}} X_{n}^{2^{n}}=X_{n}^{2^{n+1}} \subset X_{n+1}^{2^{n+1}}=Y_{n+1}$.

Assuming that $G$ is Bergman, we conclude that $G=Y_{n}=X_{n}^{2^{n}}$ for some $n \in \omega$.

By induction, we shall prove that $\varphi\left(X_{n}^{k}\right) \subset[-n k, n k]$ for $k \in \omega$. For $k=0$, we have $\varphi\left(X_{n}^{0}\right)=$ $\varphi(\{e\})=\{0\}=[-n 0, n 0]$. Assume that for some $k \in \omega$ we have proven that $\varphi\left(X_{n}^{k}\right) \subset[-n k, n k]$. 
Taking into account that for every $g \in X_{n}$ and $x \in G$ we have $\varphi(g x) \subset \varphi(x)+\left[-n_{\{g\}}, n_{\{g\}}\right] \subset$ $\varphi(x)+[-n, n]$, we conclude that $\varphi\left(X_{n} x\right) \subset \varphi(x)+[-n, n]$ and hence,

$$
\varphi\left(X_{n}^{k+1}\right)=\varphi\left(X_{n} \cdot X_{n}^{k}\right) \subset \varphi\left(X_{n}^{k}\right)+[-n, n] \subset[-n k, n k]+[-n, n] \subset[-n(k+1), n(k+1)] .
$$

Then $\varphi(G)=\varphi\left(X_{n}^{2^{n}}\right) \subset\left[-n 2^{n}, n 2^{n}\right]$, which means that the function $\varphi$ is bounded.

Finally, we prove that $(4) \Rightarrow(3)$. Assume that $G$ is almost Shelah and $\operatorname{cf}(|G|)>\omega$. Assume that $G=\bigcup_{n \in \omega} X_{n}$ for some increasing sequence of subsets such that $X_{n}=X_{n}^{-1}$ and $X_{n} \cdot X_{n} \subset$ $X_{n+1}$ for all $n \in \omega$. By induction we can show that $X_{n}^{2^{k}} \subset X_{n+k}$ for every $k \in \omega$.

Since $\operatorname{cf}(|G|)>\omega$, there exists $n \in \omega$ such that $\left|X_{n}\right|=|G|$. Since $G$ is almost Shelah, $G=X_{n}^{2^{m}} \subset X_{n+m}$ for some $m \in \omega$ and hence $X_{n+m}=G$, which contradicts the choice of the sequence $\left(X_{k}\right)_{k \in \omega}$.

Corollary 2. The finitary ballean of the permutation group $S_{X}$ of any set $X$ is mu-bounded.

Question 7. Is every so-function on a Bergman group (in particular, on the group $S_{X}$ ) constant at infinity?

Problem 1. Find the largest $n$ for which every n-Shelah group is finite.

Remark 3. Answering the problem [24], Yves Cornulier proved that each 3-Shelah group is finite.

Author Contributions: The principal ideas of this paper belong to Igor Protasov who wrote an initial draft; Taras Banakh improved the proofs of some theorems and made the final editing of the paper.

Funding: This research received no external funding.

Conflicts of Interest: The authors declare no conflict of interest.

\section{References}

1. Cornulier, Y.; de la Harpe, P. Metric Geometry of Locally Compact Groups; EMS Tracts in Mathematics; European Mathematical Society: Zürich, Switzerland, 2016.

2. De la Harpe, P. Topics in Geometric Group Theory; University Chicago Press: Chicago, IL, USA, 2000.

3. Dranishnikov, A.N.; Ferri, S. On the Higson-Roe corona. Russ. Math. Surv. 1997, 52, 1017-1028. [CrossRef]

4. Protasov, I.V. Coronas of balleans. Topol. Appl. 2005, 149, 149-160. [CrossRef]

5. Protasov, I.; Zarichnyi, M. General Asymptology; VNTL Publ.: Lviv, Ukraine, 2007.

6. Roe, J. Lectures on Coarse Geometry; Univ. Lecture Ser. 31; American Mathematical Society: Providence, RI, USA, 2003.

7. Bergman, G.M. Generating infinite symmetric groups. Bull. Lond. Math. Soc. 2006, 38, 429-440. [CrossRef]

8. Shelah, S. On a problem of Kurosh, Jónsson groups, and applications. In Word Problems, II, Conference on Decision Problems in Algebra, Oxford, 1976; Studies in Logic and the Foundations of Mathematics 95; North-Holland Publishing Company: Amsterdam, The Netherlands, 1980; pp. 373-394.

9. Dranishnikov, A.N. Asymptotic topology. Russ. Math. Surv. 2000, 55, 1085-1129. [CrossRef]

10. Dydak, J.; Hoffland, C. An alternative definition of coarse structures. Topol. Appl. 2008, 155, 1013-1021. [CrossRef]

11. Protasov, I.; Banakh, T. Ball Stuctures and Colorings of Graphs and Groups; VNTL Publ.: Lviv, Ukraine, 2003; 148p.

12. Protasov, I.V. A note on bornologies. Mat. Stud. 2018, 49, 13-18. [CrossRef]

13. Jech, T. Set Theory; Springer: Berlin, Germany, 2003. 
14. Banakh, T.; Protasov, I. The Normality and Bounded Growth of Balleans. Available online: https: / / arxiv.org/abs/1810.07979 (accessed on 18 October 2018).

15. Banakh, T.; Higes, J.; Zarichnyi, I. The coarse classification of countable abelian groups. Trans. Amer. Math. Soc. 2010, 362, 4755-4780. [CrossRef]

16. Banakh, T.; Protasov, I.; Repovs, D.; Slobodianiuk, S. Classifying homogeneous cellular ordinal balleans up to coarse equivalence. Colloq. Math. 2017, 149, 211-224. [CrossRef]

17. Protasov, I.; Slobodianiuk, S. On asymorphisms of groups. J. Group Theory 2017, 20, 393-399. [CrossRef]

18. Protasov, I.V. Normal ball structures. Math. Stud. 2003, 20, 3-16.

19. Filali, M.; Protasov, I.V. Slowly oscillating functions on locally compact groups. Appl. Gen. Topol. 2005, 6, 67-77.

20. Protasov, I.; Slobodianiuk, S. A note on partitions of groups. Quest. Answ. Gen. Topol. 2015, 33, 61-70.

21. Droste, M.; Holland, W.C. Generating automorphism groups of chains. Forum Math. 2005, 17, 699-710. [CrossRef]

22. Droste, M.; Truss, J. The uncountable cofinality of the automorphism group of the countable universal distributive lattice. Demonstr. Math. 2011, 44, 473-479. [CrossRef]

23. Tolstykh, V. On Bergman's property for the automorphism groups of relatively free groups. J. Lond. Math. Soc. 2006, 73, 669-680. [CrossRef]

24. Banakh, T. A Shelah Group in ZFC. Available online: https://mathoverflow.net/questions/313516 (accessed on 22.10.2018).

(C) 2019 by the authors. Licensee MDPI, Basel, Switzerland. This article is an open access article distributed under the terms and conditions of the Creative Commons Attribution (CC BY) license (http://creativecommons.org/licenses/by/4.0/). 